\title{
Erreichbarkeiten und Einwohnerpotenziale Zentraler Orte
}

Ein Ansatz aus Thüringen

\section{Accessibilities and Population Potentials of "Central Places"}

An approach from Thuringia

\section{Kurzfassung}

Im Zuge der Fortschreibung des Landesentwicklungsplanes Thüringen wurde vor dem Hintergrund sinkender Bevölkerungszahlen und verbesserter Erreichbarkeitverhältnisse eine Überarbeitung des Zentrale-Orte-Konzeptes von 1993 veranlasst. Mithilfe von Erreichbarkeitsanalysen im motorisierten Individualverkehr wurden dafür die künftigen Einwohnerpotenziale der Zentralen Orte höherer Stufe berechnet, um so die landesplanerische Bedeutung dieser Orte abzuschätzen. Unter Berücksichtigung der derzeitigen Ausstattungs- und Auslastungsmerkmale konnten darauf aufbauend Empfehlungen für ein künftiges System Zentraler Orte getroffen werden, das versucht, zwischen flächendeckender Versorgung und wirtschaftlicher Tragfähigkeit zu vermitteln. Die dabei offengelegten derzeitigen und künftigen Erreichbarkeitsdefizite liefern wichtige Hinweise für ergänzende strukturpolitische Maßnahmen des Landes.

\begin{abstract}
In the course of up-dating the State Development Plan for the federal state of Thuringia, and against the backdrop of dwindling populations and improvements in accessibility, the decision was taken to overhaul the "central-place"concept introduced in 1993. Drawing on accessibility analyses based on private-car use, the future population potentials of so-called "higher-order centres" have been recalculated in order to gauge the long-term significance of such centres for spatial planning within the state. In the light of current levels of infrastructure endowment and capacity utilisation, the authors are able to proceed to formulating recommendations for a future system of "central places " which strives to mediate between blanket supply and economic viability. The current and anticipated shortcomings thus revealed in respect of accessibility provide important pointers toward the types of supplementary structure-policy measures which the state will need to adopt in the future.
\end{abstract}

\section{Geänderte Rahmenbedingungen der zentralörtlichen Festlegungen in Thüringen}

Zurzeit wird der Landesentwicklungsplan Thüringen aus dem Jahr $1993^{1}$ grundsätzlich überarbeitet. Diese Überarbeitung schließt auch die nach $₫ 9$ Thüringer Landesplanungsgesetz vorgesehenen Festlegungen zu Grundzügen der Siedlungsentwicklung und Zentralen Orten mit ein.
Die im Jahr 1993 getroffenen Festlegungen zu Zentralen Orten höherer Stufe müssen insbesondere aus folgenden Gründen überprüft werden:

- Die Bevölkerungsprognosen zeigen, dass Thüringen insgesamt in Zukunft mit einem deutlichen Bevölkerungsrückgang zu rechnen hat. Diesen Bevölke- 
rungsveränderungen, die regional differenziert stattfinden werden, ist bei der Ausweisung Zentraler Orte Rechnung zu tragen.

- Das Verkehrsnetz in Thüringen hat seit 1993 wesentliche Veränderungen erfahren, die auch derzeit noch nicht abgeschlossen sind. Insbesondere im motorisierten Individualverkehr kommt es dadurch zu einer deutlichen Veränderung der Erreichbarkeitsverhältnisse. Diese tendenzielle Vergrößerung der zentralörtlichen Einzugsbereiche ist bei der künftigen Ausweisung Zentraler Orte zu berücksichtigen.

- Im künftigen System der Landesplanung sollen nur noch Oberzentren und Mittelzentren ausgewiesen werden. Die Ausweisung von Grundzentren obliegt der Regionalplanung. Die bisherigen Zwischenstufen sollen entfallen. ${ }^{2}$

Vor diesem Hintergrund wurde dem Lehrgebiet Verkehrspolitik und Raumplanung an der Fachhochschule Erfurt der Auftrag erteilt, eine Überprüfung der zentralörtlichen Ausweisungen des LEP 1993 hinsichtlich der künftigen Tragfähigkeit vorzunehmen und Empfehlungen für ein aus Versorgungssicht optimiertes zentralörtliches System zu treffen. ${ }^{3}$ Der nachfolgende Aufsatz dokumentiert das methodische Vorgehen, einen beispielhaften Auszug der vorgenommenen Berechnungen und Überprüfungen sowie die getroffenen Empfehlungen.

\section{Das Zentrale-Orte-Konzept (ZOK) als Instrument der Raumordnung}

Seit Jahren wird über den planerischen Sinn und Unsinn, über die Wirksamkeit und Wirkungslosigkeit, über die Zukunftsfähigkeit und Rückwärtsgewandtheit des Zentrale-Orte-Konzeptes als Instrument der Raumordnung diskutiert. Diese Diskussion soll und kann an dieser Stelle nicht wiederholt werden, zumal mit dem unlängst erschienenen Band der $\mathrm{ARL}^{4}$ „Das Zentrale-Orte-Konzept “ eine grundlegende Aufarbeitung dieser Diskussion stattgefunden hat.

Wenn auch - im Sinne einer Erfolgskontrolle - die Wirksamkeit des Zentrale-Orte-Konzepts (ZOK) nicht im Detail nachgewiesen werden konnte, wird es im Ergebnis dieser Diskussion doch großenteils als unverzichtbarer Baustein der Landes- und Regionalplanung angesehen, da es ein grundsätzlich sinnvolles und koordinierendes Grundgerüst für raumordnerische Ziele und regionalpolitische Entwicklungsmaßnahmen liefern kann. Leitvorstellung des ZOK ist dabei die Bündelung und Konzentration von Versorgungsfunktionen und Nachfragpotenzialen in bestimmten Orten, um insbesondere in Flächenländern ${ }^{5}$
- eine überörtliche Versorgung sicher zu stellen,

- die wirtschaftliche Tragfähigkeit der Versorgung zu gewährleisten,

- leistungsfähige Standorte für Wohnen und Arbeiten in allen Teilräumen zu erhalten und zu entwickeln sowie

- die dezentrale Konzentration der Siedlungsstruktur zu bewahren.

Die planerische Relevanz erfährt das ZOK durch die Kopplung konkreter Zielaussagen und raumwirksamer Maßnahmen an zentralörtliche Funktionszuweisungen, wie sie in Abbildung 1 dargestellt sind. Besonders die Konzentration der Siedlungstätigkeit sowie des (großflächigen) Einzelhandels auf die Zentralen Orte findet sich als raumordnerische Zielaussage in zahlreichen Raumordnungsplänen ${ }^{6}$; zentralörtliche Ausweisungen bilden häufig ein Entscheidungskriterium für die Gestaltung von Verkehrsangeboten?; mit der $\mathrm{Zu}$ weisung ökonomischer Entwicklungsfunktionen an zentrale Orte können Förderprogramme verknüpft werden; auch werden Zentrale Orte in der Regel bei den Mittelzuweisungen der Länder besser gestellt als die sonstigen Gemeinden. ${ }^{8}$

Abbildung 1

Raumwirksamkeit des Zentrale-Orte-Konzepts

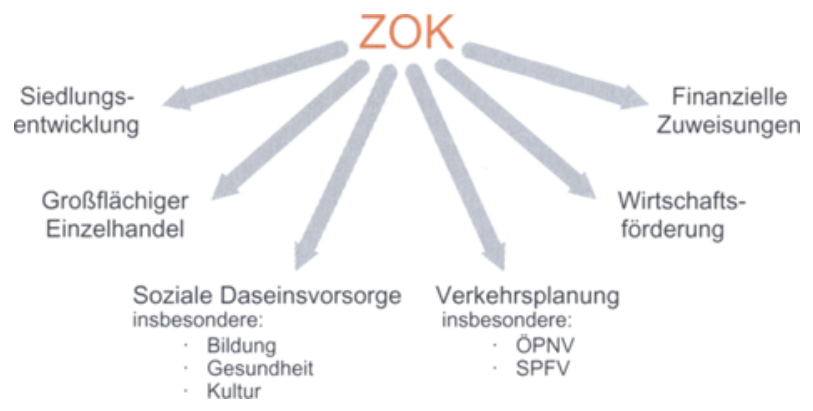

Quelle: Gather 2002

Gleichwohl hat sich dieses "generalistische" Prinzip, eine Vielzahl von Maßnahmen an zentralörtliche Funktionszuweisungen zu koppeln, oft als nur wenig wirksam erwiesen und erfährt in der Regel zahlreiche Modifikationen $^{9}$ wie die Konzentration der Ausweisung von Siedlungsschwerpunkten entlang von SPNVAchsen, die Ausweisung von gewerblichen Schwerpunktorten oder Fremdenverkehrsorten u.ä. Die lebhaften und leidenschaftlich geführten Diskussionen auf kommunaler Ebene zeigen jedoch, welche Bedeutung der Ausweisung als Zentralem Ort in der politischen Praxis zugemessen wird. Um diese Funk- 
tionszuweisung nicht allein dem daraus häufig resultierenden „opportunistischen Prinzip“"10 folgen zu lassen, ist es erforderlich, für die landesplanerischen Festlegungen der Zentralen Orte eine auf nachvollziehbaren Kriterien beruhende Diskussionsgrundlage zu liefern.

\section{Zielgrößen und Schwellenwerte im System Zentraler Orte}

Die Ausweisung Zentraler Orte höherer Stufe hat zwischen zwei grundsätzlichen und in der Praxis gegenläufigen Leitvorstellungen $\mathrm{zu}$ vermitteln.

(1)

Flächendeckende Versorgung in allen Landesteilen mit zentralörtlichen Funktionen innerhalb der Zumutbarkeit (Daseinsvorsorge)

(2)

Sinnvolle Bündelung und Konzentration zentralörtlicher Funktionen in leistungsfähigen und sich selbst tragenden Wirtschafts- und Verwaltungszentren (Sicherung der Tragfähigkeit)

Die Abstimmung des immanenten Zielkonflikts dieser Leitvorstellungen ist im Einzelfall schwierig: Eine sehr große Anzahl Zentraler Orte verspricht nominell die flächendeckende Versorgung mit zentralen Gütern und eine Minimierung des Verkehrsaufwandes, doch kann hierdurch die Tragfähigkeit der Zentralen Orte im Einzelfall in Gefahr geraten. Die Konzentration auf wenige Zentrale Orte dagegen mag die Leistungsfähigkeit dieser Zentren sicherstellen, doch kann es hierbei $\mathrm{zu}$ erheblichen Versorgungslücken in Randlagen der Versorgungsbereiche kommen.

Sowohl für die anzustrebenden Erreichbarkeiten, die einen zumutbaren Versorgungsbereich definieren, als auch für die Ausstattungsmerkmale und MindestgröBen Zentraler Orte, die Auskunft über die wirtschaftliche Tragfähigkeit liefern sollen, liegen Zielgrößen und Orientierungswerte vor, die die Grundlagen für die nachfolgenden Untersuchungen bildeten.

\subsection{Erreichbarkeiten}

Im Sinne der Daseinsvorsorge sowie einer Gleichwertigkeit der Lebensbedingungen ist eine ausreichende Versorgung der Bevölkerung mit zentralen Gütern sicherzustellen. Entscheidend sind hierfür Erreichbarkeitsstandards, die Zielgrößen hinsichtlich einer zumutbaren Reisezeit in den Zentralen Ort aus seinem Verflechtungsbereich angeben. Die ARL hat in ihrer grundlegenden Studie ${ }^{11}$ folgende Erreichbarkeitsstandards für den ÖPNV aufgestellt:
Reisezeiten ins Oberzentrum: Reisezeiten ins Mittelzentrum: Reisezeiten ins Grundzentrum:

max. 90 Minuten max. 60 Minuten max. 30 Minuten

In der hier für Thüringen vorgelegten Untersuchung wurden Reisezeiten im motorisierten Individualverkehr (MIV) zugrunde gelegt. Die Betrachtung von Reisezeiten im MIV wurde gewählt, da $90 \%$ der Wege im ländlichen Raum mit diesem Verkehrsmittel zurückgelegt werden ${ }^{12}$ und über die Reisezeiten im MIV auch eine mögliche Angebotsgestaltung im ÖPNV hinreichend abschätzbar ist. ${ }^{13}$ Die Erreichbarkeitsstandards, die für die nachfolgenden Empfehlungen von Thüringer Mittelzentren angelegt wurden, sind in Tabelle 1 dokumentiert.

Tabelle 1

Zugrunde gelegte Erreichbarkeitsstandards im motorisierten Individualverkehr

$\begin{array}{llr}\text { Oberzentrum } & \text { Zielwert } & 60 \text { Minuten } \\ & \text { Zumutbarkeit } & 90 \text { Minuten } \\ \text { Mittelzentrum } & \text { Zielwert } & \text { 30 Minuten } \\ & \text { Zumutbarkeit } & \text { 45 Minuten } \\ & \text { (angrenzende Bundesländer: 30 Minuten) }\end{array}$

Bei einem Überschreiten der Zumutbarkeitsgrenzen wird davon ausgegangen, dass eine Versorgung mit zentralörtlichen Gütern in diesen Bereichen nicht mehr gewährleistet ist. Der Besuch bzw. die Versorgung aus Mittelzentren angrenzender Bundesländer wird dann für Thüringer Teilräume als zumutbar erachtet, wenn eine Erreichbarkeit von bis zu 30 Minuten im MIV gegeben ist.

\subsection{Ausstattungsmerkmale}

Ausstattungsmerkmale geben Hinweise darauf, welche zentralen Güter und Dienstleistungen in den jeweiligen zentralörtlichen Verflechtungsbereichen für eine ausreichende Versorgung der Bevölkerung vorgehalten werden sollten. Die nachfolgende Übersicht zeigt die Empfehlungen der ARL für die hier untersuchten Ober- und Mittelzentren.

Diese Ausstattungsmerkmale sind lediglich Richtgrößen, die ein idealtypisches (Mindest-)angebot abbilden; sie liefern aber bereits einen wesentlichen Hinweis darauf, dass z. T. erhebliche Einwohnerpotenziale erforderlich sind, um eine wirtschaftliche Auslastung dieser zentralen Güter und Dienstleistungen zu gewährleisten. Insbesondere bei den privatwirtschaftlich organisierten Bereichen (Handel und Dienstleistungen) muss diesem Aspekt besondere Beachtung zuteil 
Tabelle 2

Strategische Größen und Ausstattungsmerkmale von Ober- und Mittelzentren ${ }^{14}$

\begin{tabular}{|c|c|c|}
\hline $\begin{array}{l}\text { Zentralitäts- } \\
\text { stufen }\end{array}$ & $\begin{array}{l}\text { Strategische Größen (in den Handlungsfeldern Versorgung, } \\
\text { Verkehr, Siedlung, Wirtschaft) }\end{array}$ & $\begin{array}{l}\text { Instrumentelle Ansatzpunkte/ } \\
\text { Beispiele für Konkretisierungen }\end{array}$ \\
\hline Oberzentren & $\begin{array}{l}\text { Wissenscluster von nationaler und regionaler Bedeutung } \\
\text { Private und öffentliche Steuerungs- und Dienstleistungs- } \\
\text { funktionen. Spezialisierter Arbeitsmarkt mit regionalen und } \\
\text { nationalen Verflechtungen } \\
\text { Hochwertige Kultur- und Freizeitangebote/Standorte der } \\
\text { Bündelung verschiedener kultureller Funktionen } \\
\text { Hochwertige Verkehrsinfrastruktur } \\
\text { Standort räumlich-funktionaler Arbeitsteilung in einer } \\
\text { polyzentrischen Siedlungsstruktur }\end{array}$ & $\begin{array}{l}\text { Hochschule, } \\
\text { Einrichtungen des Innovationstransfers } \\
\text { Sitz einiger Großunternehmen } \\
\text { Spezialisierte Dienstleistungsunternehmen } \\
\text { Sitz regionaler Behörden } \\
\text { Theater mit ständigem Ensemble, } \\
\text { Regelmäßige saisonale Veranstaltungen der Kultur und } \\
\text { Unterhaltung (Kultursommer usw.) } \\
\text { Ausdifferenzierte Formen von Alternativkultur } \\
\text { Güterverkehrszentrum, Güterverteilzentrum, } \\
\text { ICE/IC-Bahnhof } \\
\text { Großstadt-„City“ mit Warenhäusern Neue Zentrentypen (z.B. } \\
\text { Shopping Malls) Größere Stadtteilzentren }\end{array}$ \\
\hline Mittelzentren & $\begin{array}{l}\text { Regionale Bildungs- und Ausbildungsfunktion } \\
\text { Arbeitsmarktzentrum } \\
\text { Zentrale Funktionen für Kultur, Freizeit und } \\
\text { Unterhaltung } \\
\text { ÜberregionaleVerkehrsanbindung } \\
\text { Zentrale Einzelhandels- und Dienstleistungsfunktion } \\
\text { Einrichtungen des Gesundheitswesens } \\
\text { Kompakte Stadt der kurzen Wege }\end{array}$ & $\begin{array}{l}\text { Schulen der Sekundarstufe II } \\
\text { Sonderschule } \\
\text { Öffentliche Bibliothek } \\
\text { Veranstaltungshalle, Öffentliches Schwimmbad, weitere } \\
\text { wohnungsnahe Freizeiteinrichtungen } \\
\text { Anbindung an Schienenfernverkehr } \\
\text { ÖPNV-Knotenpunkt } \\
\text { Stadtzentrum mit breit gefächertem Einzelhandels- und } \\
\text { Dienstleistungsangebot, Fußgängerzone } \\
\text { Regelkrankenhaus, Fachärzte } \\
\text { Konzentration der Bautätigkeit auf Siedlungsschwerpunkte mit } \\
\text { ÖPNV-Erschließung, (Inter)kommunale Gewerbegebiete }\end{array}$ \\
\hline
\end{tabular}

werden. Dies gilt zunehmend aber auch für die Bereitstellung ehemals oder derzeit noch öffentlicher Dienstleitungen wie Gesundheitswesen und öffentlicher Personenverkehr, Post und Telekommunikation, die sich ebenfalls stärker an Auslastungszahlen als am Ziel einer Daseinsvorsorge orientieren werden.

\subsection{Größe von Zentralen Orten und Einzugsbereiche}

Für die wirtschaftliche Tragfähigkeit der zentralen Güter ist die Nutzungsintensität von entscheidender Bedeutung. Besonders zentrale Güter des höheren Bedarfs, wie sie in Mittel- und Oberzentren angeboten werden sollten, sind dabei auf ein ausreichendes Einwohner- und ein daraus abgeleitetes Nachfragepotenzial angewiesen.

Vor dem Hintergrund der Orientierungswerte der $A R L^{15}$ für Bevölkerungszahlen in Zentralen Orten und ihren Verflechtungsbereichen wurde für die Untersuchung der Thüringer Mittelzentren ein modifizierter Ansatz gewählt, wie er in Tabelle 3 dokumentiert ist.

\section{Tabelle 3}

Einstufungssystematik von Mittelzentren unter Berücksichtigung von Trägfähigkeitskriterien

\begin{tabular}{|c|c|c|c|}
\hline \multirow{2}{*}{$\begin{array}{c}\text { Inneres Einwohner- } \\
\text { potenzial } \\
\text { (30 Min. im MIV) } \\
\end{array}$} & \multicolumn{3}{|c|}{ Einwohner im ZO 2020} \\
\hline & 5 bis 10 Tsd. & 10 bis 20 Tsd. & $>20 \mathrm{Tsd}$ \\
\hline 0 bis 30 Tsd. & $\begin{array}{c}\text { Keine Tragfähig- } \\
\text { keit als } M Z \\
\text { zu erwarten } \\
\end{array}$ & $\begin{array}{l}\text { Erforderlich- } \\
\text { keitsprüfung }\end{array}$ & $\begin{array}{c}\text { Tragfähigkeit } \\
\text { als MZ } \\
\text { wahrscheinlich } \\
\end{array}$ \\
\hline 30 bis 50 Tsd. & $\begin{array}{l}\text { Erforderlich- } \\
\text { keitsprüfung }\end{array}$ & $\begin{array}{c}\text { Tragfähigkeit } \\
\text { als MZ } \\
\text { wahrscheinlich }\end{array}$ & $\begin{array}{l}\text { Tragfähigkeit } \\
\text { als MZ gegeben }\end{array}$ \\
\hline$>50$ Tsd. & $\begin{array}{l}\text { Tragfähigkeit } \\
\text { als MZ } \\
\text { wahrscheinlich }\end{array}$ & $\begin{array}{l}\text { Tragfähigkeit } \\
\text { als } \mathrm{MZ} \text { gegeben }\end{array}$ & $\begin{array}{l}\text { Tragfähigkeit } \\
\text { als } M Z \text { gegeben }\end{array}$ \\
\hline
\end{tabular}

Unter Berücksichtigung der Bevölkerungsprognosen für 2020 ist demnach dann mit einer auch langfristigen Tragfähigkeit eines Mittelzentrums zu rechnen, wenn beide der von der ARL genannten Orientierungswerte (10 000 Einwohner im ZO und 30000 Einwohner im Verflechtungsbereich) erreicht werden oder aber über 20000 Einwohner im Zentralen Ort bzw. über 50000 Einwohner im inneren Einzugsbereich dieses Ortes le- 
ben. Eine Tragfähigkeit als Mittelzentrum ist dagegen nicht zu erwarten, wenn keiner der beiden von der ARL genannten Orientierungswerte erreicht wird. Wird nur einer der beiden Orientierungswerte erreicht, ist eine Detailüberprüfung im Einzelfall erforderlich.

\section{Methodisches Vorgehen}

Ausgehend von den Zielgrößen für die zentralörtliche Versorgung in Thüringen wurde für die vorliegende Untersuchung das in Abbildung 2 gezeigte methodische Vorgehen gewählt. Dieses hier dokumentierte Vorgehen bezieht sich allein auf die Ausweisung von Mittelzentren, doch ist der methodische Ansatz grund- sätzlich auf zentralörtliche Funktionszuweisungen jeder Stufe übertragbar.

\subsection{Ermittlung von Einzugsbereichen}

In einem ersten Arbeitsschritt erfolgte die Ermittlung von Einzugsbereichen der 35 im LEP 1993 aufgeführten Zentralen Orte höherer Stufe in Thüringen aufgrund der Isochronen im übergeordneten Straßennetz 2020. Entsprechend den oben getroffenen Zielvorgaben wurden diese Berechnungen für 30 und $45 \mathrm{Minu-}$ ten (MZ) sowie 60 Minuten (OZ) durchgeführt. Die Berechnungen erfolgten EDV gestützt unter Berücksichtigung der Neubauvorhaben im Bundesautobahn-
Abbildung 2 Methodisches Vorgehen
Ermittlung von Einzugsbereichen der derzeit ausgewiesenen 35 Zentralen Orte

höherer Stufe in Thüringen im übergeordneten Straßennetz 2020

- 30 Min im MIV

- 45 Min im MIV

Bevölkerungsprognose 2020 und

Berechnung von Einwohnerpotenzialen der 35 ausgewiesenen Zentralen Orte

höherer Stufe in Thüringen aufgrund der o.g. Isochronen

Weist der ZO > 10000 Einwohner oder ein inneres Einwohnerpotenzial ( 30 Min.) von > 30000 Einwohnern auf?

Nein

$$
\text { Ja }
$$

Tragfähigkeitsprüfung II: Weist der $Z O>10000$ Einwohner und ein inneres Potenzial (30 Min.) von > 30000 Einwohnern auf?

Beträgt der innere Einzugsbereich ( $30 \mathrm{Min}$ ) $>50000$

Einwohner oder leben im ZO > 20000 Einwohner?

Nein

Erforderlichkeitsprüfung und Optimierung Verfügt der $Z O$ bereits über eine ausreichende zentralörtliche Ausstattung?

Ist ohne den Ort eine zumutbare Versorgung großer Teilräume

in Thüringen nicht gewährleistet?

Gibt es keine zumutbare Versorgung aus Zentralen Orten

benachbarter Bundesländer?

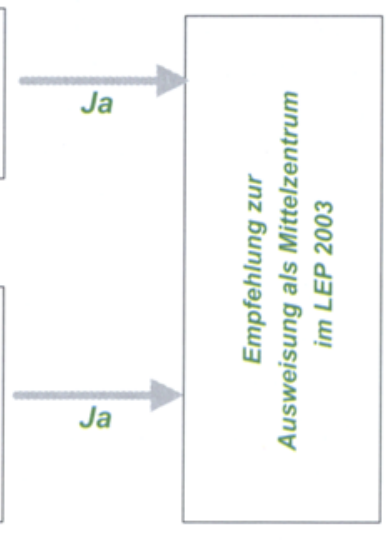

Nein

Die Überprüfung des zentralen Ortes zeigt, dass aufgrund der Größe des Ortes oder infolge einer ausreichenden Versorgung aus besser geeigneten Orten die langfristige ökonomische Tragfähigkeit als Mittelzentrum nicht gegeben ist. 
netz unter Zugrundelegung der in Tabelle 4 angegebenen Reisegeschwindigkeiten.

Tabelle 4

Reisegeschwindigkeit 2020 (Modellannahmen)

\begin{tabular}{|l|c|}
\hline Straßentyp & Mittlere Reisegeschwindigkeit \\
\hline BAB & $100 \mathrm{~km} / \mathrm{h}$ \\
Bundesstraße außerorts & $60 \mathrm{~km} / \mathrm{h}$ \\
Sonstige Straßen außerorts & $50 \mathrm{~km} / \mathrm{h}$ \\
Ortsdurchfahrten & $30 \mathrm{~km} / \mathrm{h}$ \\
Gemeindestraßen & $20 \mathrm{~km} / \mathrm{h}$ \\
\hline
\end{tabular}

Die Modellannahmen wurden aufgrund von Richtlinien der FGSV ${ }^{16}$ sowie von eigenen Messfahrten und verkehrsstatistischen Erhebungen im Thüringer Autobahnnet ${ }^{17}$ getroffen. Die zugrunde gelegten Streckengeschwindigkeiten befinden sich an der Untergrenze der im Regelfall realisierbaren Geschwindigkeiten, um eine regelmäßige Erreichbarkeit unabhängig von Fahrstil und Verkehrszuständen abzubilden. Die Überprüfung der Modellannahmen ergab, dass innerhalb der ermittelten Isochronen in über $90 \%$ der Fälle die tatsächliche Erreichbarkeit aus den Einzugsbereichen gegeben ist.

\subsection{Ermittlung von Einwohnerpotenzialen}

Die darauf aufbauende Berechnung von Einwohnerpotenzialen der 35 ausgewiesenen Zentralen Orte höherer Stufe in Thüringen erfolgte aufgrund der o. g. Isochronendarstellung. Grundlage der Einwohnerzahlen war die Umlegung der 9. koordinierten Bevölkerungsvorausberechnung ${ }^{18}$ auf die einzelnen Städte und Gemeinden. Die Umlegung der auf Kreisebene vorliegenden Prognosezahlen erfolgte gleichmäßig auf die einzelnen kreisangehörigen Städte und Gemeinden. Daraus evtl. resultierende Unschärfen können durch die Betrachtung von Verflechtungsbereichen weitgehend ausgeglichen werden.

\subsection{Das Konzept des inneren Einwohnerpotenzials}

Innerhalb von 30 Minuten ist mit einer Reisegeschwindigkeit im MIV von etwa $50 \mathrm{~km} / \mathrm{h}$ angesichts des dichten Straßennetzes von einem beliebigen Punkt etwa eine Fläche von $1500-2000 \mathrm{~km}^{2} \mathrm{zu}$ erreichen. Bei einer durchschnittlichen Siedlungsdichte von $150 \mathrm{E} /$ $\mathrm{km}^{2}$ in Thüringen bedeutet dies, dass jeder Zentrale Ort für rund 250000 Einwohner innerhalb von 30 Minuten erreichbar ist. Eine solche Zahl kann aber keine Beurteilungsgrundlage für die Einwohnerpotenziale im Versorgungsbereich von möglichen Mittelzentren sein.
Für die vorliegende Untersuchungen wurde daher der Ansatz des inneren Einwohnerpotenzials entwickelt. Als inneres Einwohnerpotenzial eines Zentralen Ortes wird dabei die Anzahl derjenigen Einwohner verstanden, die innerhalb der zugrundegelegten Zeit (30, 45 oder 60 Minuten) lediglich diesen Ort erreichen können. Dieser Wert steigt also mit zunehmender Entfernung der Zentralen Orte voneinander, während er in sich überlagernden Verflechtungsbereichen gegen Null tendiert.

Das innere Einwohnerpotenzial ist somit vor allem für kleinere Orte eine gute Maßzahl für die Umlandbedeutung bzw. Entbehrlichkeit von Zentralen Orten besonders in dünnbesiedelten Räumen. Das tatsächlich erreichte und versorgte Einwohnerpotenzial wird insbesondere bei großen Zentralen Orten aber deutlich über diesem Wert liegen.

\subsection{Tragfähigkeitsprüfung}

In der anschließenden Tragfähigkeitsprüfung wurde die langfristige Tragfähigkeit einer Ausweisung als Zentraler Ort überprüft. Entsprechend den Zielgrößen der Tabelle 4 scheiden dabei zunächst solche Orte aus, deren Einwohnerzahl 2020 im Zentralen Ort unter 10000 Einwohnern liegt und die über ein inneres Einwohnerpotenzial innerhalb von 30 Minuten von unter 30000 Einwohnern verfügen. Liegen mehrere dieser Orte mit sich überschneidenden Einzugsbereichen nebeneinander, ist es erforderlich, die nunmehr veränderten inneren Einwohnerpotenziale jeweils neu zu berechnen, da es hier zu einer deutlichen Erhöhung der zentralörtlichen Bedeutung kommen kann bzw. bei Nichtbeachtung beträchtliche Lücken im System zentraler Orte entstehen können.

In den darauffolgenden Bedeutungsprüfungen wurde für solche Orte die Ausweisung als Mittelzentrum empfohlen, die die in Tabelle 3 dokumentierten Orientierungswerte erfüllen und somit die langfristige Tragfähigkeit als MZ wahrscheinlich erscheinen lassen.

\subsection{Detailuntersuchung im Einzelfall}

Für diejenigen Orte und Verflechtungsbereiche, die weder unter der Tragfähigkeitsschwelle liegen noch ihre zentralörtliche Tragfähigkeit klar erkennen lassen, erfolgte abschließend eine Erforderlichkeitsprüfung und Optimierung im Einzelfall. Grundlage für diese Detailuntersuchung waren die derzeitigen Ausstattungs- und Auslastungsmerkmale ${ }^{19}$, die Berücksichtigung von Zumutbarkeitsgrenzen der Erreichbarkeit sowie die Betrachtung von Zentren auch benachbarter Bundesländer. 
5 Ergebnisse der Überprüfung: Mittelzentren und ihre Einzugsbereiche

\subsection{Künftige Erreichbarkeiten und} Einwohnerpotenziale der derzeitigen Zentralen Orte höherer Stufe

Karte 1 dokumentiert die Zentralen Orte höherer Stufe des LEP 1993 und ihre Einzugsbereiche im MIV unter Berücksichtigung der geplanten oder im Bau befindlichen Bundesfernstraßen. Hier zeigt sich, dass es insbesondere entlang der Bundesautobahnen in Mittel- und Ostthüringen, aber auch im Raum Suhl sowie zwischen Nordhausen und Heiligenstadt zur Überlagerung mehrer zentralörtlicher Einzugsbereiche kommt.
Ebenso veranschaulicht diese Karte die „inneren“ Einwohnerpotenziale, d.h. diejenigen Bereiche, die lediglich durch einen Zentralen Ort im LEP 1993 bedient werden und die Grundlage für die anschließende Beurteilung liefern. Tabelle 5 gibt eine Übersicht über die Einwohnerzahlen der untersuchten Zentralen Orte sowie ihre inneren Einwohnerpotenziale.

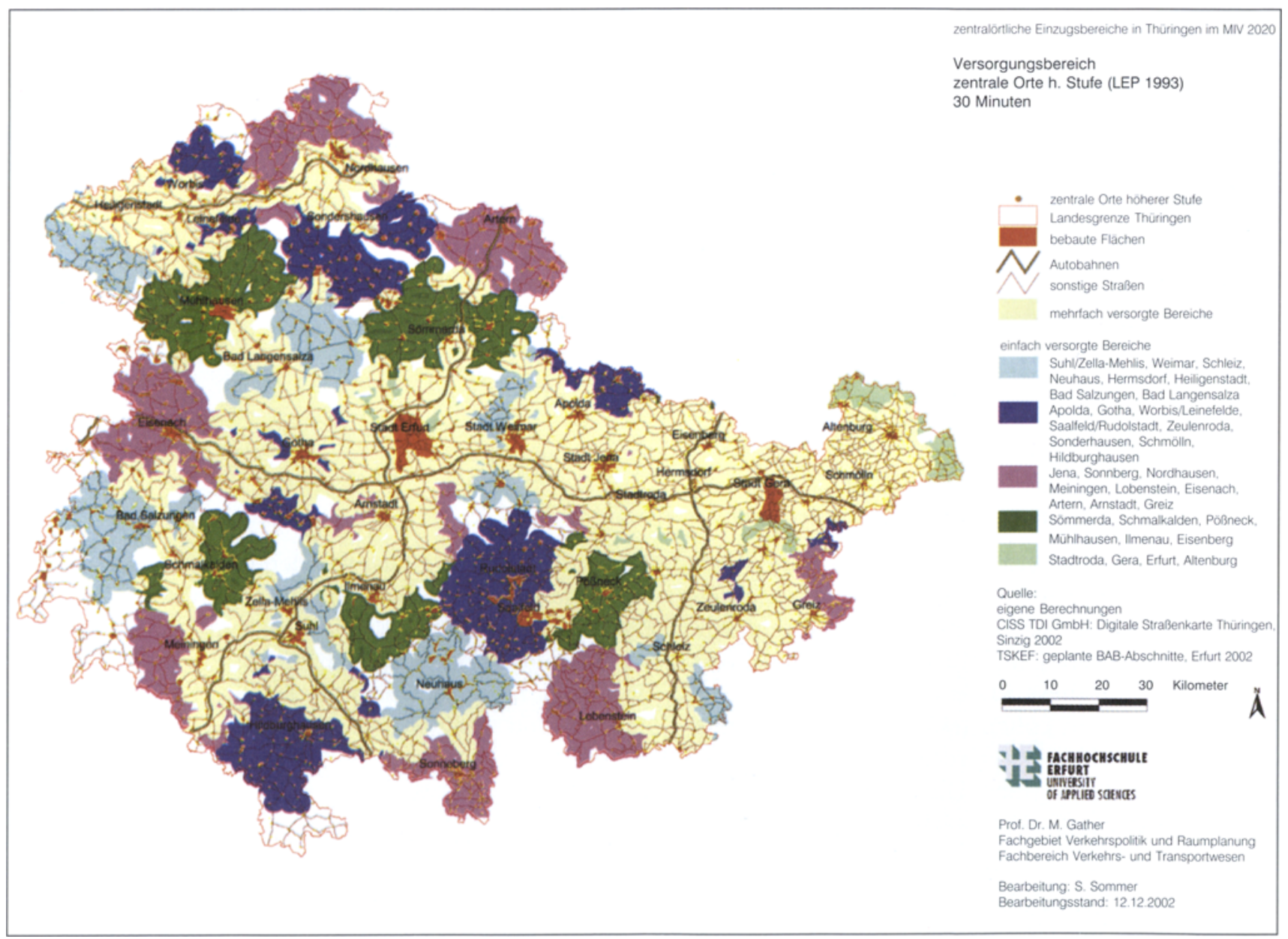




\begin{tabular}{|c|c|c|c|c|}
\hline Stadt & $\begin{array}{c}\text { Funktion } \\
\text { nach LEP '93 }\end{array}$ & $\begin{array}{l}\text { Einwohner } \\
\text { im ZO } 2020\end{array}$ & $\begin{array}{c}\text { Inneres } \\
\text { Einwohner- } \\
\text { potenzial } \\
\text { (30 Min.) }\end{array}$ & $\begin{array}{l}\text { Inneres } \\
\text { Einwohner- } \\
\text { potenzial } \\
(45 \mathrm{Min} .)\end{array}$ \\
\hline Altenburg & $\mathrm{MZ} / \mathrm{OZ}$ & 35398 & 12320 & 0 \\
\hline Apolda & $\mathrm{MZ}$ & 27665 & 8931 & 0 \\
\hline Arnstadt & $\mathrm{MZ}$ & 26477 & 1682 & 0 \\
\hline Artern & $\mathrm{MZ}$ & 6226 & 37184 & 10542 \\
\hline Bad Langensalza & $\mathrm{t} M Z$ & 18173 & 14287 & 0 \\
\hline Bad Salzungen & MZ & 15896 & 46512 & 14676 \\
\hline Eisenach & $\mathrm{MZ} / \mathrm{OZ}$ & 38100 & 63152 & 5573 \\
\hline Eisenberg & $\mathrm{MZ}$ & 12057 & 20 & 0 \\
\hline Erfurt & $\mathrm{OZ}$ & 151100 & 1553 & 0 \\
\hline Gera & $\mathrm{OZ}$ & 80500 & 10133 & 0 \\
\hline Gotha & MZ & 48942 & 14446 & 0 \\
\hline Greiz & $\mathrm{MZ}$ & 24737 & 8980 & 0 \\
\hline Heiligenstadt & $\mathrm{MZ}$ & 16728 & 9651 & 1065 \\
\hline Hermsdorf & tMZ & 9367 & 0 & 0 \\
\hline Hildburghausen & $\mathrm{MZ}$ & 12409 & 30057 & 9912 \\
\hline Ilmenau & $\mathrm{MZ}$ & 26527 & 19995 & 0 \\
\hline Jena & $\mathrm{OZ}$ & 80200 & 745 & 0 \\
\hline Lobenstein & $\mathrm{tMZ}$ & 6782 & 14967 & 2303 \\
\hline Meiningen & $\mathrm{MZ}$ & 20584 & 7748 & 1410 \\
\hline Mühlhausen & $\mathrm{MZ}$ & 35388 & 52702 & 449 \\
\hline Neuhaus & tMZ & 5831 & 27485 & 104 \\
\hline Nordhausen & $\mathrm{MZ} / \mathrm{OZ}$ & 41730 & 24888 & 1581 \\
\hline Pößneck & $\mathrm{MZ}$ & 13333 & 26549 & 0 \\
\hline Saalfeld/Rudolstadt & $\mathrm{MZ} / \mathrm{OZ}$ & 49215 & 57416 & 236 \\
\hline Schleiz & MZ & 8638 & 2050 & 0 \\
\hline Schmalkalden & $\mathrm{MZ}$ & 17226 & 29164 & 16 \\
\hline Schmölln & $\mathrm{tMZ}$ & 11279 & 1672 & 0 \\
\hline Sömmerda & $\mathrm{MZ}$ & 21634 & 30342 & 0 \\
\hline Sondershausen & $\mathrm{MZ}$ & 21041 & 20938 & 0 \\
\hline Sonneberg & $\mathrm{MZ}$ & 21848 & 34469 & 1010 \\
\hline Stadtroda & $\mathrm{tMZ}$ & 6842 & 33 & 0 \\
\hline Suhl/Zella-Mehlis & $\mathrm{MZ} / \mathrm{OZ}$ & 49932 & 547 & 0 \\
\hline Weimar & $\mathrm{MZ} / \mathrm{OZ}$ & 56800 & 18404 & 0 \\
\hline Worbis/Leinefelde & $\mathrm{MZ}$ & 19918 & 11542 & 1184 \\
\hline Zeulenroda & $\mathrm{t} M Z$ & 13790 & 545 & 0 \\
\hline
\end{tabular}

Tabelle 5

Einwohnerzahlen und mittelzentrale Einzugsbereiche der untersuchten Zentralen Orte höherer Stufe

\subsection{Beispielhafte Detailuntersuchung mittelzentraler Teilräume: Südostthüringen}

Für den Raum Südostthüringen konnte aufgrund der in Kapitel 3 dokumentierten Tragfähigkeitskriterien im ersten Arbeitsschritt kein Mittelzentrum empfohlen werden. Die Städte Pößneck und Zeulenroda erfüllen diese Kriterien nur teilweise. Die Städte Schleiz und Lobenstein erfüllen keines der Tragfähigkeitskriterien, wurden aber wegen sich überlagernder Einzugsbereiche in die folgende Detailuntersuchung für Südost-Thüringen mit aufgenommen.

Karte 2 veranschaulicht die Einzugsbereiche der untersuchten Zentralen Orte sowie der angrenzenden Mittelzentren im Ostthüringer Raum. Tabelle 6 dokumentiert die wichtigsten Berechnungsergebnisse der Detailuntersuchung für die genannten Orte.
Insgesamt zeigt sich, dass von den untersuchten Zentren aufgrund der Einwohner im Zentralen Ort, des inneren Einwohnerpotenziales sowie der zentralörtlichen Ausstattung Pößneck am ehesten in der Lage ist, die Funktion eines Mittelzentrums für diesen Teilraum wahrzunehmen und somit als Mittelzentrum empfohlen werden konnte.

Als ungeeignet für die künftige Ausweisung als Mittelzentrum wurden dagegen die Städte Lobenstein und Zeulenroda aus folgenden Gründen angesehen:

- Bad Lobenstein hat nur eine sehr geringe Einwohnerzahl im Zentralen Ort und verfügt nur über ein unzureichendes zentralörtliches Angebot. Teile des berechneten inneren Einwohnerpotenzials können von Pößneck abgedeckt werden. Trotz eines theoretisch immer noch großen inneren Einwohnerpotenzials ist die langfristige Tragfähigkeit eines Mittelzentrums Lobenstein daher nicht gegeben. 
Karte 2

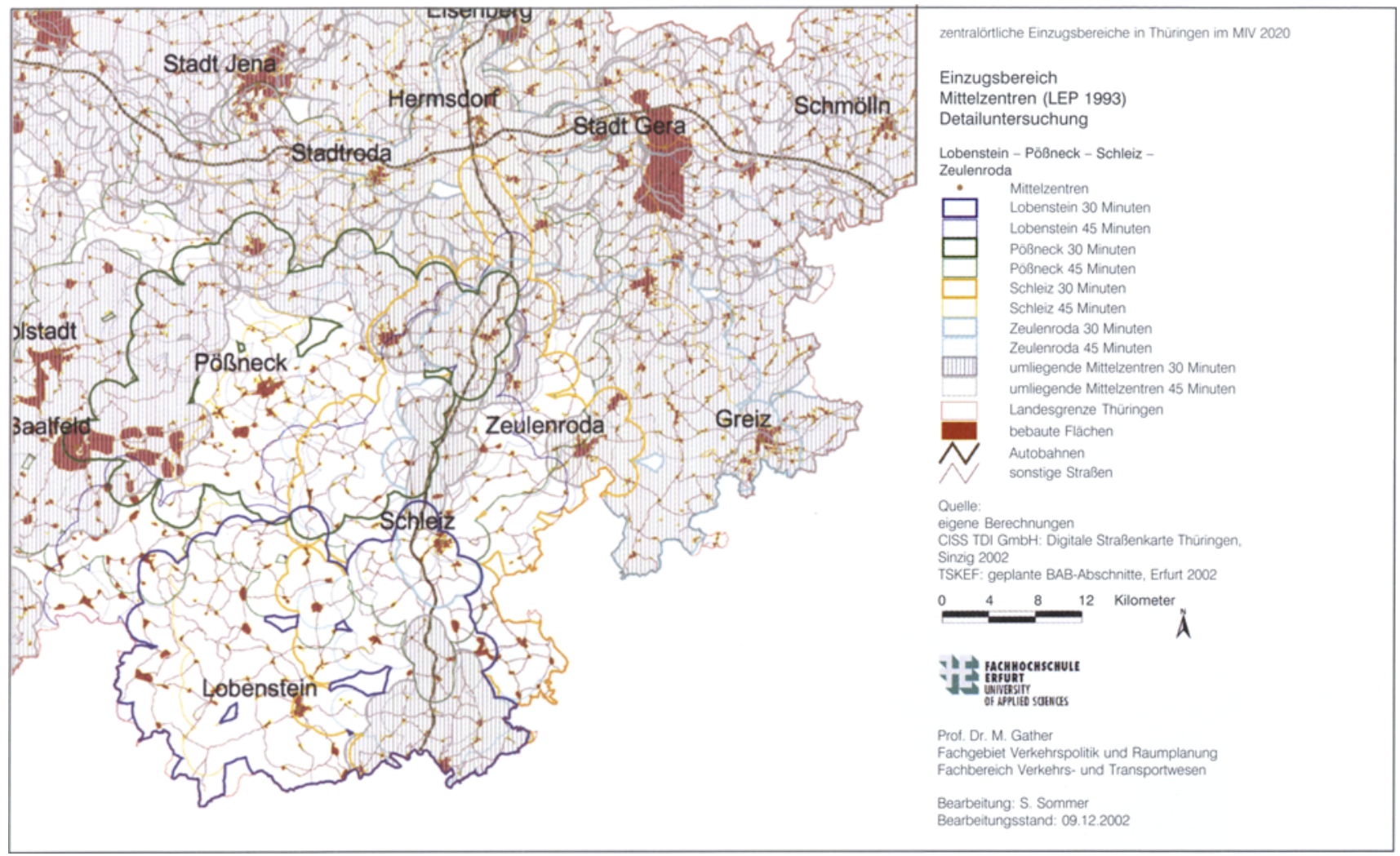

Tabelle 6

Detailuntersuchung Südostthüringen ${ }^{20}$

\begin{tabular}{|c|c|c|c|c|}
\hline Stadt & Lobenstein & Pößneck & Schleiz & Zeulenroda \\
\hline Eimwohner im Zentralen Ort 2020 & 6782 & 13333 & 8638 & 13790 \\
\hline Innerer Versorgungsbereich $30 \mathrm{Min}$. (ohne die mituntersuchte ZO) & 35360 & 30833 & 33192 & 14814 \\
\hline Innerer Versorgungsbereich $45 \mathrm{Min}$. (inkl. Hof in Bayern) & 24562 & 4626 & 26403 & 7189 \\
\hline Sitz der Kreisverwaltung & Außenstelle & Außenstelle & Hauptsitz & Außenstelle \\
\hline Schulen der Sekundarstufe II & 1 & 1 & 1 & 2 \\
\hline Sonderschule & 2 & 2 & 2 & 1 \\
\hline Öffentliche Bibliothek & Ja & ja & ja & ja \\
\hline Offentliches Hallenbad & Kurbad & ja & nein & ja \\
\hline Anbindung an Schienenfernverkehr & Nein & nein & nein & nein \\
\hline SPNV-Anbindung & Ja & ja & ja & $\mathrm{ja}$ \\
\hline ÖPNV-Knotenpunkt & Regionalbus & $\begin{array}{c}\text { Stadtbus/ } \\
\text { Regionalbus }\end{array}$ & $\begin{array}{c}\text { Stadtbus } \\
\text { Regionalbus }\end{array}$ & $\begin{array}{c}\text { Stadtbus/ } \\
\text { Regionsbus }\end{array}$ \\
\hline Einzelhandelsumsatz (Mio DM 1996)"1 & 58,1 & 222,4 & 189,7 & 185,2 \\
\hline Geldinstitute (Anzahl) & 2 & 3 & 3 & 3 \\
\hline Regelkrankenhaus & ja & ja & ja & nein \\
\hline Niedergelassene Ärzte (Anzahl) & 22 & ca. 50 & ca. 35 & 42 \\
\hline Kommunale Gewerbegebiete (ha) & 7,4 & 51,58 & 59,53 & 41,91 \\
\hline
\end{tabular}


- Zeulenroda liegt an der Untergrenze der empfohlenen Einwohnerzahl im Zentralen Ort. Die Stadt besitzt aufgrund ihrer relativen Nähe zu leistungsfähigeren Mittelzentren keinen ausgeprägten eigenen Einzugsbereich und weist teilweise Defizite in der zentralörtlichen Ausstattung auf. Aufgrund seiner geografischen Lage könnte Zeulenroda auch bei einem ausreichenden Angebot die Versorgungssituation in Ostthüringen nicht nachhaltig verbessern. Die Ausweisung von Zeulenroda als Mittelzentren wurde daher nicht empfohlen.

Die Kreisstadt Schleiz erfüllt die meisten der in Kapitel 3.2 formulierten Anforderungen an die zentralörtlichen Ausstattungsmerkmale, weist aber ein nur geringes Einwohnerpotenzial im Zentralen Ort auf. Aufgrund der Lage an der leistungsfähigen A 9 ist die Erreichbarkeit von Schleiz im MIV als außerordentlich gut anzusehen, so dass ein entsprechendes mittelzentrales Angebot innerhalb einer zumutbaren Zeit von 45 Minuten auch aus dem Raum Lobenstein durch über 25000 Einwohner wahrgenommen werden kann, die ansonsten über keine zumutbare mittelzentrale
Versorgung verfügen würden. Trotz der geringen Einwohnerzahl im Zentralen Ort wurde aufgrund der zentralen Lage in einem ansonsten großräumig unterversorgten Gebiet die Stärkung mittelzentraler Funktionen mit der Ausweisung eines Mittelzentrums Schleiz empfohlen.

\section{Empfehlungen für ein zentralörtliches System in Thüringen}

Karte 3 zeigt die zusammenfassenden Empfehlungen für ein System Zentraler Orte höherer Stufe in Thüringen. Dieses vorgeschlagene System versucht, zwischen einer optimalen räumlichen Versorgung aller Landesteile unter Einbeziehung der zukünftigen Straßeninfrastruktur und einer dauerhaften wirtschaftlichen Tragfähigkeit der Angebote unter Berücksichtigung der künftigen Bevölkerungsentwicklung zu vermitteln. Es zeigt aber auch auf, in welchen Landesteilen Erreichbarkeitsdefizite und daraus drohende Versorgungsschwächen vorliegen werden

Karte 3

Empfehlung zentrale Orte

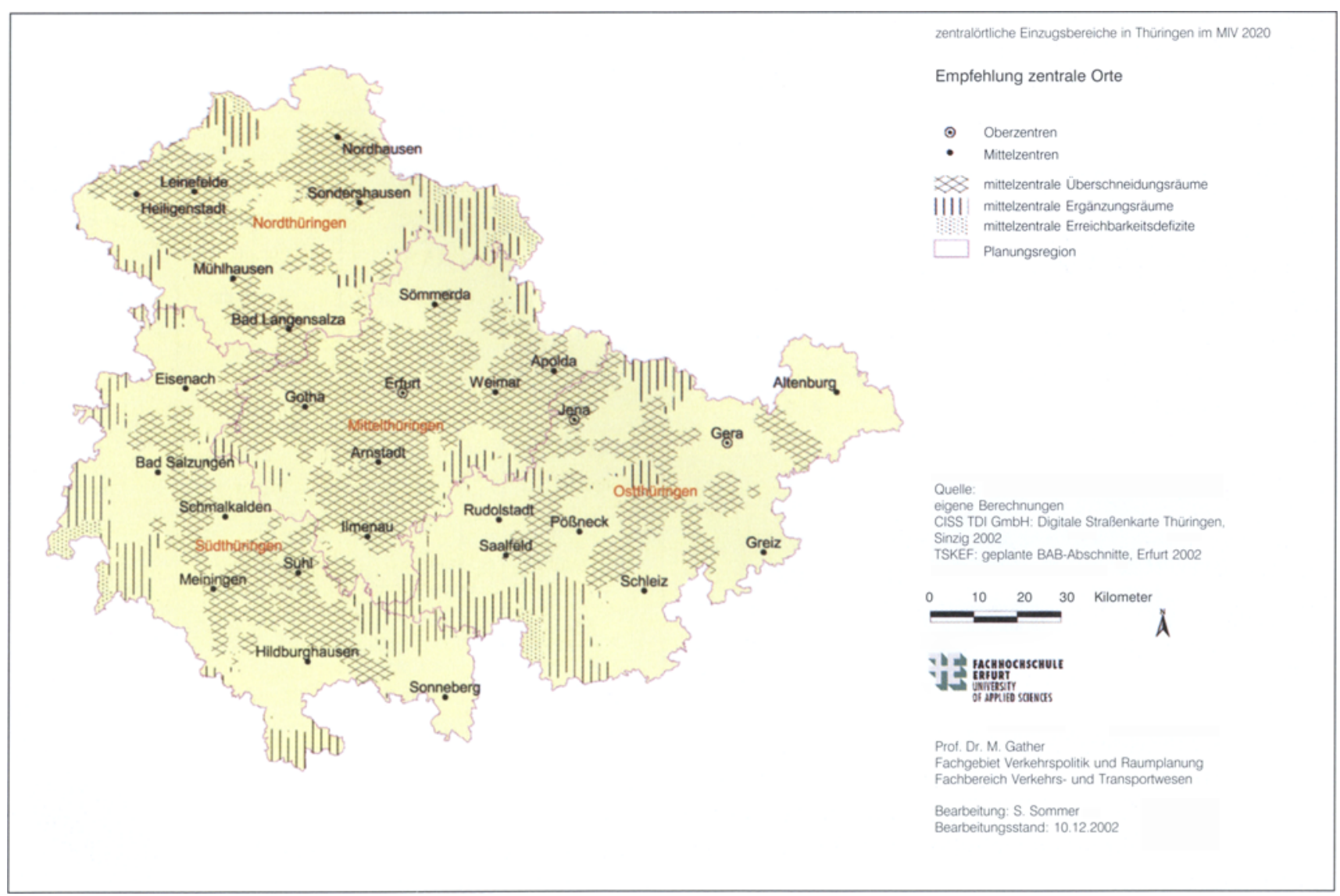


Durch die empfohlenen Oberzentren Erfurt, Jena und Gera kann eine oberzentrale Versorgung in Mittel- und Ostthüringen sichergestellt werden. Die Ausweisung weiterer Oberzentren wurde nicht empfohlen, da die untersuchten Städte Nordhausen und Suhl aufgrund ihrer derzeitigen Bedeutung und Ausstattung nicht in der Lage sein werden, ein umfassendes leistungsfähiges oberzentrales Angebot vor allem im privatwirtschaftlichen Bereich bereitzustellen. Auch unter Berücksichtung der oberzentralen Versorgung aus angrenzenden Bundesländern werden daher Teile Südwest- und Nordthüringens weiterhin Lücken in der Erreichbarkeit eines Oberzentrums aufweisen. Hier sollte bei der Fachplanung dafür Sorge getragen werden, dass bestehende Angebote hochwertiger Dienstleistungen in den Regionen weiter beibehalten bzw. ergänzt werden.

Durch die 24 vorgeschlagenen Mittelzentren ist eine gute Erreichbarkeit mittelzentraler Angebote für den Großteil der Bevölkerung Thüringens (über $90 \%$ der Einwohner) gewährieistet. In zahlreichen Teilräumen führt die Dichte von Zentralen Orten höherer Stufe zu einer deutlichen Überlagerung von Einzugsbereichen. Diese Bereiche sind als „mittelzentrale Überschneidungsräume" gekennzeichnet, in denen auch bei einer Funktionsteilung zwischen den betroffenen Orten eine ausreichende Versorgung sichergestellt werden kann. Entlang der „Thüringer Städtekette“, in Saalfeld/Rudolstadt sowie in Südthüringen sollten diese Kooperationsmöglichkeiten für ergänzende oberzentrale Angebote genutzt werden.

„Mittelzentrale Ergänzungsräume" mit schwächerer mittelzentraler Versorgung sind dadurch gekennzeichnet, dass relativ große Entfernungen (bis zu $45 \mathrm{~min}$ ) in das nächste Mittelzentrum zurückgelegt werden müssen. In diesen Gebieten besonders des Thüringer Waldes (Neuhaus a.R.), der Rhön, im Raum Lobenstein sowie in Nordost-Thüringen (Artern) leben etwa 7 bis $8 \%$ der Einwohner Thüringens, die auch nur zum geringen Teil durch Mittelzentren aus benachbarten Bundesländern (v.a. Sangerhausen und Eschwege) versorgt werden können. Hieraus resultieren zum einen besondere Anforderungen an einen leistungsfähigen ÖPNV in den Zentralen Ort, um die räumlichen Nachteile zu kompensieren. Zum anderen besteht in diesen Teilräumen die Erforderlichkeit der Vorhaltung ausgewählter mittelzentraler Angebote (besonders Schulen und Krankenhäuser) in dafür besonders geeigneten Grundzentren, um eine ausreichende Versorgung der Bevölkerung sicher zu stellen. Hierdurch kann auch die Versorgungslage der angrenzenden Räume "mittelzentraler Erreichbarkeitsdefizite" entscheidend verbessert werden.
In den Räumen „mittelzentraler Erreichbarkeitsdefizite" schließlich ist eine zumutbare Erreichbarkeit des nächsten Mittelzentrums (bis zu 45 min im MIV) nicht gegeben, selbst die Vorhaltung eines grundzentralen Angebotes kann sich im Einzelfall als problematisch erweisen. In diesen Teilräumen in den Randlagen Südwest- und Ostthüringens leben unter $1 \%$ der Gesamtbevölkerung. Hier können teilweise leistungsfähige Grundzentren in den benachbarten „Mittelzentralen Ergänzungsräumen“ wichtige Versorgungsaufgaben übernehmen; darüber hinaus sollte seitens der Landesplanung auf die Fachplanungen hingewirkt werden, auch bei schwacher Auslastung für eine wohnortnahe Versorgung der Bevölkerung Sorge zu tragen sowie die Landkreise ebenso wie kleinere Gemeinden mit besonderen finanziellen Anreizen bei der Übernahme von interkommunalen Aufgaben und Angeboten zu unterstützen.

\section{Anmerkungen}

(1)

LEP 1993

(2)

Im derzeitigen System der Landesplanung existieren auf höherer Stufe noch die Zwischenstufen „MZ mit Teilfunktionen eines $O Z$ “, „teilfunktionales Mittelzentrum" sowie auf unterer Stufe „Unterzentren“ und „Kleinzentren“.

(3)

Die Studie wurde im Dezember 2002 abgeschlossen (Gather; Sommer 2002) und steht auch als Down-load zur Verfügung (www.fh-erfurt.de/vt/projekte).

(4)

Blotevogel 2002

(5)

So z.B. LRP-SH 1998, S. 52

(6)

Vgl. BBR 2002

(7)

So unlängst der Ad-hoc-Arbeitskreis der ARL „Netzstruktur der DB AG aus Sicht der Raumordnung“ (2002) zu dem überregional bedeutsamen Schienennetz [vgl. Heinze (2002)]

(8)

Vgl. von Rohr 2002

(9)

Bzw. ist als "universales Siedlungsstrukturkonzept obsolet" (Deiters 1996, S. 631) geworden und sollte durch raumstrukturell angepasste Konzepte ergänzt werden. 
(10)

Gerosko 2002, S. 33 ff.

(11)

Blotevogel 2002, S. 302

(12)

Hautzinger; Pfeiffert; Tasaux-Becker 1994

(13)

Dies gilt insbesondere für periphere ländliche Räume, in denen mit einer Zunahme flexibler Bedienformen (Anruf-SammelTaxi, Anrufbus o.ä.) gerechnet werden kann.

(14)

Nach Blotevogel 2002, S. 247

(15)

Quelle: Blotevogel 2002, S. 298

(16)

FGSV 1988, S. 15

(17)

Pfannenstill 2002

(18)

TLS 2000

(19)

Hier konnte auf eine umfangreiche Studie (IWR 1997) zurückgegriffen werden, die bereits 1997 für die Landesplanung in Thüringen erarbeitet worden war.

(20)

Quelle: Eigene Berechnungen und Erhebungen

(21)

Quelle: IWR 1997 Anlagenband III

\section{Literatur}

Blotevogel, Hans H. (Hrsg.) (2002): Fortentwicklung des Zentrale-Orte-Konzeptes. Forschungs- und Sitzungsberichte der ARL Band 217. - Hannover

Bundesamt für Bauwesen und Raumordnung (Hrsg.) (2002): Beispiele der Anwendung des Zentrale-Orte-Konzepts in den Bundesländern. Bearbeitet von Doris Pick und Gerhard Stiens. -Bonn

CISS TDI GmbH (2002): Digitale Straßenkarte Thüringen. - Sinzig

Deiters, Jürgen (1996): Die Zentrale-Orte-Konzeption auf dem Prüfstand. Wiederbelebung eines klassischen Raumordnungsinstruments? In: Inform. z. Raumentwickl., H. 10, S. 631-646
Forschungsgesellschaft für Straßen- und Verkehrswesen, Arbeitsgruppe Verkehrsplanung (1998); Richtlinien für die Anlage von Straßen RAS. Teil: Leitfaden für die funktionale Gliederung des Straßennetzes RAS-N. Ausgabe 1988. - Köln

Gather, Matthias; Sommer, Sebastian (2002): Berechnung Zentralörtlicher Einzugsbereiche 2020 und Empfehlungen für ein System Zentraler Orte in Thüringen. Im Auftrag der Thüringer Staatskanzlei, Abt. Landesplanung. Fachhochschule Erfurt. - Erfurt

Genosko, Joachim (2002): Politik und Zentrale-Orte-System. In: Blotevogel, S. 31-36

Hahne, Ulf; von Rohr, Götz (1999): Ansatzpunkte zur Weiterentwicklung des Zentrale-Orte-Systems Schleswig-Holsteins. In: Raumforschung und Raumordnung, H. 2/3, S. 143-154

Hautzinger, Heinz; Pfeiffer, Manfred; Tassaux-Becker, Brigitte (1994): Mobilität. Ursachen, Meinungen, Gestaltbarkeit. - Heilbronn

Heinze, G.W. u.a. (2002): Überregional bedeutsames Schienennetz in Deutschland aus raumordnerischer Sicht. In: Raumforschung und Raumordnung, H. 5/6, S. 291 ff.

Institut für Wirtschaftsförderung und Regionalmarketing (Hrsg.) (1997): Die Zentralen Orte Thüringens und ihre Funktionsteilung. Im Auftrag des Thüringer Ministeriums für Wirtschaft und Infrastruktur, Abt. Raumordnung und Landesplanung. - Leipzig

Landesentwicklungsprogramm vom 10. November 1993 (1993). GVBI. Für den Freistaat Thüringen Nr. 34

Landesraumordnungsprogramm Schleswig-Holstein (1998). - Kiel

Pfannerstill, Elmar (2002): Reisezeiten im Straßennetz. Laufendes Projekt am Fachbereich Verkehrs- und Transportwesen der Fachhochschule Erfurt. Zwischenbericht 2002. - Erfurt

Thüringer Landesamt für Statistik (2000): Ergebnisse der Vorausberechnung nach Landkreisen und kreisfreien Städten bis 2020 aufgrund der 9. koordinierten Bevölkerungsvorausberechnung. - Erfurt

von Rohr, Götz; Buhren, Karin (2002): Zentrale Orte und kommunaler Finanzausgleich - ein Ländervergleich. In: Blotevogel (2002), S. 43-54

Prof. Dr. Matthias Gather

Fachgebiet Verkehrspolitik und Raumplanung

Fachbereich Verkehrs- und Transportwesen

Fachhochschule Erfurt

Postfach 101363

99013 Erfurt

E-Mail: gather@verkehr.fh-erfurt.de 